\title{
Disseminated Bacillus Calmette-Guerin Infection
}

National Cancer Institute

\section{Source}

National Cancer Institute. Disseminated Bacillus Calmette-Guerin Infection. NCI

Thesaurus. Code C113669.

A rare systemic and life-threatening infection associated with vaccination with the live attenuated strain of Mycobacterium bovis, bacillus Calmette-Guerin. 